\section{X-ray Star Problems Defined}

by our Cosmology Correspondent

AT it specialist meeting of the Royal Astronomical Society on January 12, problems raised by the discovery of $\mathrm{X}$-ray sources in binary systems were placed in the perspective of the latest observations at radio, optical and X-ray wavelengths. Few detailed answers to these problems have yet emerged; but theoreticians are now learning which questions must be answered by the observations in order to differentiate between those models which are at present plausible.

The first speaker, Dr K. A. Pounds (University of Leicester), described some of these observations at X-ray frequencies-not, this time, the work of his own group, but chiefly the Uhuru observations. He reported that American Science and Engineering are now preparing the third Uhuru catalogue (3U) which will include some 200 sources, down to an intensity $10^{-3}$ times that of the Crab. It seems that these 200 sources are divided almost equally between galactic and extragalactic objects. Of the galactic sources, the binary systems which are arousing so much interest form a subset of six in our Galaxy and one in the Small Magellanic Cloud.

Several of these sources show a rapid variation with a period of a second or two (reminiscent of pulsars) superimposed on a binary period of hours or days. One of the most interesting results to emerge from extended observations during the past few months is that in some cases at least this rapid variation is speeding up. Her $\mathrm{X}-1$, for example, has a "pulsar" period of $1.2 \mathrm{~s}$ and a binary period of 1.7 day; the former now seems to be increasing by $4 \mu \mathrm{s}$ in six months, according to recent MIT data which Dr Pounds reported.

Two of the sources have been studied in detail by optical astronomers. $\mathrm{Dr}$ P. Murdin (Royal Greenwich Observatory) described these studies of $\mathrm{Her}$ $\mathrm{X}-1$ and Cyg X-1-which have received this concentrated attention, of course, simply because of the lack of reliable optical identifications of the other sources. Her $\mathrm{X}-1$ has been identified with $\mathrm{HZ}$ Her, a 14 mag star which shows the same binary period of 1.7 day as the $\mathrm{X}$-ray source. The primary in the system seems to be affected by the X-ray emission of the companion, but a reasonably comprehensive model has been proposed by Forman et al. (Astrophys. J. Lett., 177, L103; 1972 ; see also Nature Physical Science, 240,$169 ; 1972$ ). At X-ray frequencies, the source also shows a regular 35-day variation, which has yet to be explained, and there is marginal evidence for optical variations at the "pulsar" period.

Cyg X-1, however, is the joker in the binary X-ray star pack. As Dr Murdin put it, this is "something completely different". This optical identification was achieved by a roundabout route, which verges on being a circular argument. Early last year, the emission of the source in different X-ray channels changed abruptly; at the same time, a new radio source appeared in that part of the sky, and the radio position is good enough to permit identification of the radio source with a BO supergiant. There is no direct reason to connect the X-ray source with either the'star or radio source, but the coincidence of the sudden change in X-ray properties with the appearance of the radio source is so strong that nobody has seriously questioned the identification of Cyg X-1 with the BO supergiant. This is the source which many theorists have turned to as evidence that black holes exist. Dr Murdin stressed, however, that although the source is clearly unusual, and asymmetry in the light curve implies that the stars in the system are non-spherical, the optical data are consistent with the view that the secondary is an early main sequence star. There is no pressing need to invoke the "counsel of desperation" hypothesis that there is a black hole in the system. Black holes, he says, are a solution looking for a problem.

In discussing the radio properties of these objects, Dr G. K. Miley (Westerbork) concentrated chiefly on the remarkable outburst of Cyg X-3. Most of the work described has already appeared in Nature (see special issue of Nature Physical Science, October 23, 1972). But one interesting possibility which Dr Miley pointed to is that the triple radio source associated with Sco X-1 might have originated in an explosion similar to the event recently observed in Cyg X-3. Other than Cyg X-3 at the time of the two flares, all these binary $\mathrm{X}$-ray systems are weak or non-existent radio sources; only Cyg X-1 and Cyg X-3 are firmly identified at radio frequencies, and, although Sco X-1 may be of the same family as Cyg X-3, no one consistent model will explain the observed properties of all three objects.

It was left to Professor Martin Rees (at present at the University of Sussex but shortly to return to Cambridge as Sir Fred Hoyle's successor as Plumian Professor) to discuss the theoretical problems-not, he was quick to point out, to provide the answers to the questions raised by the observations. It seems that the overall features are happily explained by a model in which a giant star has a magnetic neutron star companion. Under those conditions, slight mass loss from the giant even a strong stellar wind-can cause enough matter to accrete onto the neutron star to produce the observed $\mathrm{X}$-rays, because as much as 10 per cent of the rest mass energy of the infalling matter is liberated as it falls down the neutron star's potential well. The mechanism is not unlike that of a pulsar in reverse, for pulsars are generally believed to be losing matter by outflow along lines of magnetic force. Professor Rees suggested that this could be why X-ray "pulsars" are seen in binary systems, where there is infalling matter, but radio pulsars are found only in isolation. Also, transfer of angular momentum by the infalling matter should cause the neutron star to spin faster, in agreement with the latest X-ray observations.

The detailed answers to the many problems raised at this meeting remain to be found. Why do the $\mathrm{X}$-rays in these sources sometimes "switch off" for days at a time? Why do the optical variations show no similar behaviour? How did these binary systems evolve to their prescnt state? Is there a black hole in Cygnus X-1? And in addition searches of survey plates show that the optical variations of Her X-1 sometimes switch off for years at a time. This raises the number of time scales associated with that source, at least, to four. But the problems are not insoluble; their number simply indicates the vigorous growth of this new branch of astronomy.

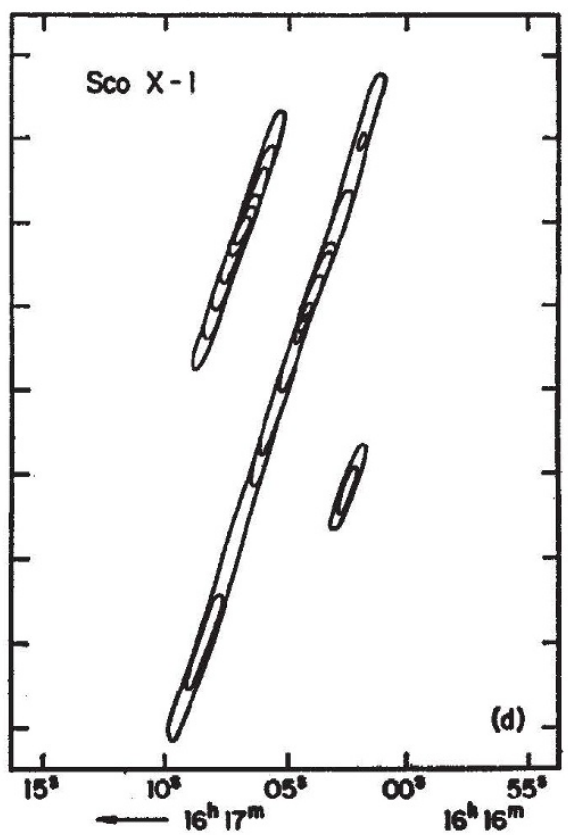

Sco X-1 mapped at 2,695 MHz (after Hjellming and Wade, Astrophys. J. Lett., 164, L1 ; 1971). Could the triple structure-smeared in this figure by the antenna response- be the result of an outburst like that recently observed in Cyg X-3? 